\title{
The use of e-cigarettes among university students in Malaysia
}

\author{
Sharifa Ezat Wan Puteh', Roslina Abdul Manap², Tidi Maharani Hassan², Izzah Syazwani Ahmad', Idayu Badilla Idris', \\ Fariza Md Sham ${ }^{3}$, Andrea Ban Yu Lin², Chun lan Soo ${ }^{2}$, Rashidi Mohamed Pakri Mohamed ${ }^{4,5}$, Ahmad Irdha Mokhtar ${ }^{3}$, \\ Hazli Zakaria ${ }^{6}$, Jing Lee ${ }^{7}$, Amer Siddiq Amer Nordin ${ }^{5}$, Suthahar Ariaratnam ${ }^{8}$, Mohd Zaliman Mohd Yusoff ${ }^{9}$
}

\begin{abstract}
INTRODUCTION E-cigarette use is an emerging phenomenon with increasing recognition and acceptance globally. This study aims to create a profile of e-cigarette users among university students in Malaysia.

METHODS The study was conducted using a cross-sectional research involving six universities in Malaysia. A semi-structured questionnaire was distributed to 1302 randomly selected students, who either smoked cigarettes and/or e-cigarettes. The 2011 version of Global Adult Tobacco Surveys (GATS) tool was used to record the respondents' sociodemographic data.

RESULTS The study revealed that $74.9 \%$ of the respondents smoked e-cigarettes; $40.3 \%$ used both cigarettes and e-cigarettes (dual users), and $34.5 \%$ were exclusive e-cigarette users. The exclusive use of e-cigarettes was related to gender $(\mathrm{OR}=0.18,95 \% \mathrm{CI}: 0.09-0.39)$. Also, male respondents were the majority users (95\%). Of the respondents, $75.2 \%$ were Malays, $98.0 \%$ single and most believed they have no health problems $(92.1 \%)$. Further findings revealed the occurrence of adverse effects, dizziness $14.4 \%$, cough $14.1 \%$, and headaches $12.4 \%$. Overall, $57.8 \%$ of the respondents used e-cigarettes as a smoking cessation tool, while others consider e-cigarettes a self-image enhancing tool or as part of social activities.

CONCLUSIONS Further research on the use of e-cigarettes should be conducted on a large number of respondents in other settings to augment the findings of this study, and also guide policy making on and prevention practice of e-cigarette use, among the general student population in Malaysia.
\end{abstract}

ABBREVIATIONS Cl: confidence interval, DASS: depression anxiety stress scales, GATS: Global Adult Tobacco Surveys, IQR: interquartile, MOHE: Ministry of Higher Education Malaysia, MYR: Malaysian Ringgit, NGO: non-governmental organization, OR: odds ratio, SPSS: Statistical Package for Social Science, USM: University of Science Malaysia, UTeM: Technical University of Malaysia, WHO: World Health Organization.

\author{
AFFILIATION \\ 1 Department of Community Health, \\ Faculty of Medicine, Universiti Kebangsaan \\ Malaysia Medical Centre, Kuala Lumpur, \\ Malaysia \\ 2 Respiratory Units, Faculty of Medicine, \\ Universiti Kebangsaan Malaysia Medical \\ Centre, Kuala Lumpur, Malaysia \\ 3 Department Da'wah and Leadership \\ Studies, Faculty of Islamic Studies, Universiti \\ Kebangsaan Malaysia, Selangor, Malaysia \\ 4 Department of Family Medicine, Faculty \\ of Medicine, Universiti Kebangsaan Malaysia \\ Medical Centre, Kuala Lumpur, Malaysia \\ 5 University Malaya Centre of Addiction \\ Sciences (UMCAS), University Malaya, Kuala \\ Lumpur, Malaysia \\ 6 Department of Psychiatry, Faculty of \\ Medicine, Universiti Kebangsaan Malaysia \\ Medical Centre, Kuala Lumpur, Malaysia \\ 7 Institute for Environment and \\ Development (LESTARI), Universiti \\ Kebangsaan Malaysia, Selangor, Malaysia \\ 8 Department of Psychological and \\ Behavioural Medicine, Faculty of Medicine, \\ Universiti Teknologi MARA (UiTM), Selayang \\ Campus, Batu Caves, Selangor, Malaysia \\ 9 Department of Software Engineering, \\ College of Information Technology, \\ Universiti Tenaga Nasional (UNITEN), \\ Kajang, Malaysia

\section{CORRESPONDENCE TO} \\ Sharifa Ezat Wan Puteh. Department of \\ Community Health, Faculty of Medicine, \\ University Kebangsaan Malaysia Medical \\ Centre, Cheras, 56000 Kuala Lumpur, Malaysia. \\ E-mail:sh_ezat@ppukm.ukm.edu.my
}

\section{KEYWORDS}

electronic cigarettes, smoking, university, students, Malaysia

Received: 2 October 2017

Revised: 25 October 2018

Accepted: 2 November 2018

\section{INTRODUCTION}

The electronic-cigarette (e-cigarette) is an electronic device, also known as vape, containing a cartridge filled with liquid nicotine and/or other chemicals, producing inhalable smoke. E-cigarette use has quickly gained popularity worldwide ${ }^{1}$, especially among current and former smokers ${ }^{2-6}$. E-cigarette manufacturers promote it as a safer, cheaper and an alternative product for 
smoking cessation. As the popularity and use increase, so is concern about public health. In 2009, Australia, Brazil, China, Uruguay ${ }^{7}$, Brunei, Cambodia, Indonesia, Singapore, Thailand, and Vietnam ${ }^{8}$ completely banned the sale and marketing of e-cigarettes; while New Zealand, United Kingdom, and other European countries allowed marketing of e-cigarettes. SouthEast Asia is one of the regions in the world with the highest number of countries ( 6 out of 11 countries in the region) that banned the use of e-cigarettes ${ }^{8}$. However, some aspects of the banned regulations in these countries need improvement ${ }^{9}$.

The International Tobacco Control Policy Evaluation Project (ITC Project) reports the use of e-cigarettes in 10 countries, through a survey conducted between 2009 and 2013. Malaysia was found to be the country with the highest prevalence of users, at $14 \%$, while other countries like Republic of Korea and Australia had 7\% each, United States 6\%, United Kingdom 4\%, Netherlands 3\%, Canada 1\%, and China $0.05 \%^{1}$. The 2015 Global Adult Tobacco Survey (GATS) conducted in the South-East Asian (SEAN) region, gave the following e-cigarette use prevalence for countries in the region: Indonesia $0.3 \%$ and Malaysia $0.8 \%$, in $2011^{10}$; Philippines $1.7 \%^{11}$ and Vietnam $0.2 \%^{12}$. Also, the Global Youth Tobacco Survey (GYTS) of 2016 showed the prevalence of e-cigarette use among students (age 13-15 years) to be $2.3 \%$ in Cambodia ${ }^{13}$ and $5.7 \%$ in Myanmar ${ }^{14}$. Noteworthy, the survey revealed a $3.2 \%$ prevalence rate for Malaysia ${ }^{1,14,15}$, which makes it the largest vaping market in the SEAN region ${ }^{16}$.

Wong et al. reported $39.9 \%$ of e-cigarette use among young students of higher institutions and $36 \%$ use among young professionals in their study conducted in the Selangor and Kuala Lumpur areas of Malaysia ${ }^{17}$. In an attempt to check the menace of e-cigarette use, the Higher-Education Minister of Malaysia announced in November 2015 a ban on e-cigarette use and tobacco smoking in universities. The ban is in effect in several Malaysian universities ${ }^{18,19}$.

E-cigarette use prevalence is high among the young, and diverse reasons for the use have been reported in several studies. Some studies state that cigarette (tobacco) smoking is an important associated factor of e-cigarette use among young people $\mathrm{e}^{20-23}$, while the older users consider it an alternative to tobacco smoking ${ }^{24,25}$. Other reasons for the use are: experimenting due to curiosity ${ }^{26-29}$, interesting flavours $^{30,31}$, 'just for fun ${ }^{29,32}$, popularity ${ }^{33}$ and 'just experimenting, ${ }^{34}$. The risk associated with e-cigarette use is said to be much less than that of tobacco smoking. If the claim is right, the harms related to tobacco smoking would be substantially reduced, with benefits for cardiovascular health ${ }^{35}$. However, more research needs to be conducted to ascertain the effects of e-cigarette use compared with tobacco smoking.

Several aspects of e-cigarette use in Malaysia were reported in various studies, but none was on the users' profile. As such, this study aims to explore the factors associated with e-cigarette use, in order to create e-cigarette users' profile based on sociodemographic distribution, source of information and supply, gender, and adverse effects, amongst other features, in six Malaysian universities.

\section{METHODS}

\section{Participants and procedure}

The study was conducted through a cross-sectional field survey research approach, from August 2016 to December 2016. Six out of sixteen universities situated in Klang Valley, Malaysia, were randomly selected; three public and three private universities. As prevalence for e-cigarette users among adults in Malaysia was $14 \%{ }^{1}$, and the total population was estimated at 204000 students, a sample size of $n=185$ (95\% Confidence Interval (CI) and 5\% significant level) from each of the selected universities was obtained using the Leslie Kish formula. A total of 1302 participants were chosen through a box-model random sampling technique.

Inclusion criteria of this study were: 1) university student, 2) age 18 years and older, and 3) student currently smoking a conventional cigarette or use of e-cigarette at the time of the study. Exclusion criteria were: 1) student on leave and/or not attending classes for three months, 2) student suspended by university, 3 ) student did not wish to participate in the study, and 4) student with cognitive disorders due to diseases like dementia, Parkinson's disease or schizophrenia.

\section{Measurements}

The questionnaire, in English and Malay, contained both closed and open-ended questions, divided into three sections: 1) sociodemographic background and current health status; 2) smoking history; and 
3) e-cigarette use. The sociodemographic section included age, gender, race/ethnicity, educational levels, nationality, marital status, total household income and health problems. Questionnaires were distributed to the participants by the lead researcher and a trained research assistant. Ethical approval for the study was granted by the Secretariat of Research and Innovation, Universiti Kebangsaan Malaysia Medical Centre (Code: FF-2016-301) and the administrative board of each participating university. Information sheets and consent forms were distributed to all students, and active consent was received from the participants. The respondents were duly informed that participation in the study was voluntary, and that their identity will remain anonymous. They were also informed that a response to a question was neither 'right' nor 'wrong'.

Questions on smoking history were adapted from the GATS 2011 with slight modification to suit the study. Participants were asked if they had ever smoked a cigarette, smoking habits of their immediate family members, age at smoking initiation, amount of smoking and type of cigarette used, among other questions. Questions on the reasons for e-cigarette use were based on 28 items and a 5 -point Likert scale ( $1=$ totally disagree, $2=$ disagree, $3=$ unsure, $4=$ agree, $5=$ totally agree). Responses were coded in two categories: low (total score lower than mean score) and high (total score higher than mean score).

\section{Statistical analysis}

The collected data were analyzed using SPSS version 23.0, using frequencies (n), percentages (\%), chisquare tests and multivariable binary logistic regression models. Level of significance was set at $p<0.05$ and regression results are presented as Adjusted Odds Ratios (AOR) with 95\% Confidence Interval (CI).

\section{RESULTS}

\section{Participants' background}

The respondents were between the ages of 18 to 40 years: males $n=1234(94.8 \%)$ and females $\mathrm{n}=68(5.2 \%)$; mean age and standard deviation of $21.15 \pm 2.55$ years; ethnic Malay $75.2 \%$, Malaysian nationals $97.9 \%$, and single $98.0 \%$. Distribution based on education level was $\mathrm{PhD} / \mathrm{DrPH} 0.6 \%$ and undergraduate $65.2 \%$. The household mean monthly income was MYR 5760.89 \pm 7411.14 , and the propotion of participants with a self-perception of being healthy was $92.1 \%$ (Table 1 ).

Table 1. Distribution of sociodemographic characteristics of respondents $(n=1302)$

\begin{tabular}{|c|c|c|}
\hline Variables & $\begin{array}{l}\text { Frequency } \\
\text { (n) }\end{array}$ & Per cent ( $\left.{ }^{\circ} \%\right)$ \\
\hline \multicolumn{3}{|l|}{ Age (years) } \\
\hline $18-20$ & 376 & 28.9 \\
\hline $21-25$ & 880 & 67.6 \\
\hline $26-30$ & 38 & 2.9 \\
\hline$>30$ & 8 & 0.6 \\
\hline \multicolumn{3}{|l|}{ Gender } \\
\hline Male & 1234 & 94.8 \\
\hline Female & 68 & 5.2 \\
\hline \multicolumn{3}{|l|}{ Ethnicity } \\
\hline Malay & 979 & 75.2 \\
\hline Chinese & 98 & 7.5 \\
\hline Indian & 162 & 12.4 \\
\hline $\begin{array}{l}\text { Others (e.g. Kedayan, Bajau, } \\
\text { Bangladesh, Indonesia) }\end{array}$ & 63 & 4.8 \\
\hline \multicolumn{3}{|l|}{ Nationality } \\
\hline Malaysian & 1275 & 97.9 \\
\hline $\begin{array}{l}\text { Others (e.g. Bangladesh, Indonesia, } \\
\text { Mexico) }\end{array}$ & 26 & 2.1 \\
\hline \multicolumn{3}{|l|}{ Marital status } \\
\hline Single/unmarried & 1276 & 98.0 \\
\hline Married & 26 & 2.0 \\
\hline \multicolumn{3}{|l|}{ Level of education } \\
\hline Diploma & 373 & 28.6 \\
\hline Degree & 849 & 65.2 \\
\hline Masters & 72 & 5.5 \\
\hline $\mathrm{PhD} / \mathrm{DrPH}$ & 8 & 0.6 \\
\hline \multicolumn{3}{|l|}{$\begin{array}{l}\text { Household income (MYR/Month), } \\
n=1116\end{array}$} \\
\hline$\leq 2000$ & 273 & 24.5 \\
\hline $2001-4000$ & 307 & 27.5 \\
\hline $4001-6000$ & 244 & 21.9 \\
\hline $6001-8000$ & 99 & 8.9 \\
\hline $8001-10000$ & 106 & 9.5 \\
\hline$\geq 10001$ & 87 & 7.8 \\
\hline \multicolumn{3}{|l|}{ Presence of comorbidity disease } \\
\hline None (healthy) & 1199 & 92.1 \\
\hline Asthma & 64 & 4.9 \\
\hline High cholesterol & 13 & 1.0 \\
\hline High blood pressure & 7 & 0.5 \\
\hline Diabetes mellitus & 2 & 0.2 \\
\hline Kidney problem & 3 & 0.2 \\
\hline Heart disease & 4 & 0.3 \\
\hline $\begin{array}{l}\text { Others (e.g Allergic, Migraine, Gastric, } \\
\text { Cancer) }\end{array}$ & 21 & 1.6 \\
\hline
\end{tabular}




\section{Smoking and e-cigarette use profile}

Within our sample population of students, $34.5 \%$ were exclusive e-cigarette users, and $40.3 \%$ were dual users, indicating that $74.8 \%$ of the respondents were current e-cigarette users (Figure 1). Moreover, $18.7 \%$ were current smokers and 6.4\% neither smoked nor used e-cigarettes. Among current e-cigarette users, 40.3\% used cigarettes and e-cigarettes simultaneously. In all, 57.5\% (749) of the respondents were introduced to e-cigarettes by colleagues in the university, $37.5 \%$
(488) by friends outside the university, 36.6\% through the internet (websites, social networking sites, blogs, and e-mails) and $28.8 \%$ through the communication media (television, radio, mail, print, billboards and outdoor advertisement). A propotion of $37.9 \%$ of the respondents bought e-cigarette products from retailers, $17.6 \%$ from their fellow university students, and $14.8 \%$ online.

Findings in this study revealed that most of the e-cigarette users preferred locally produced e-liquid

Figure 1. Cigarette/e-cigarette users among students (n=1302)

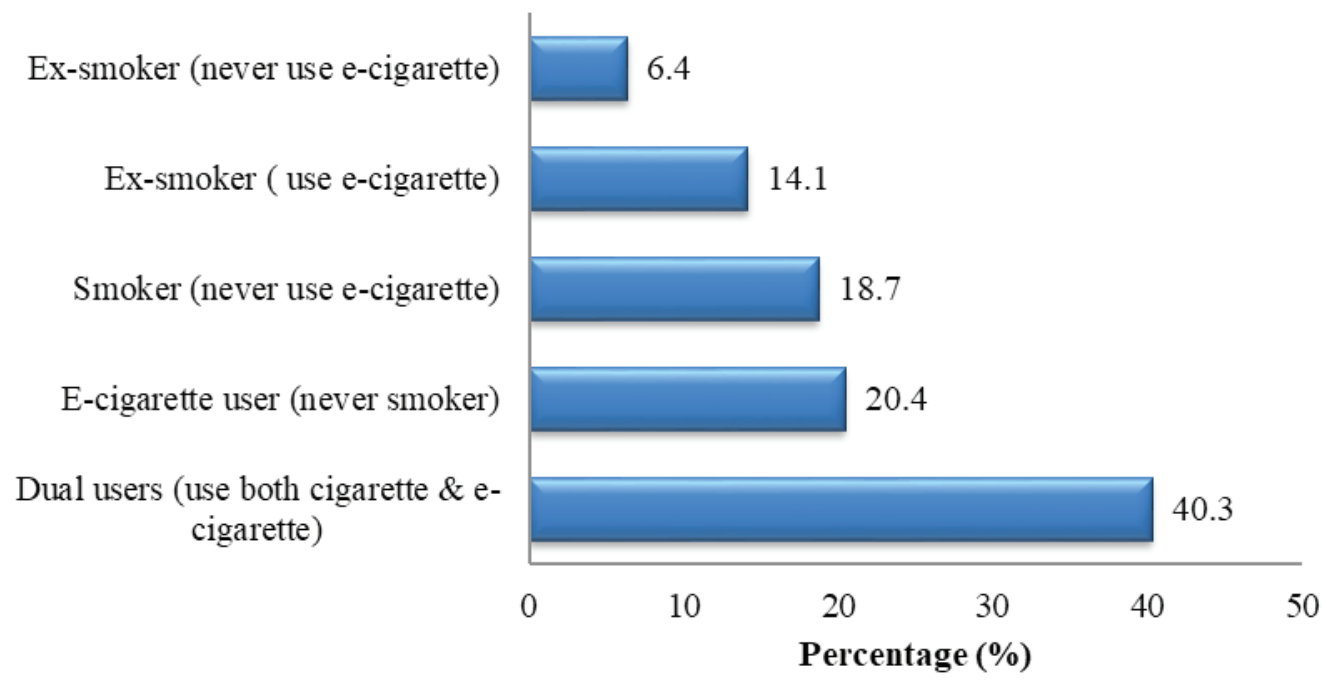

(55.6\%) rather than imported e-liquids (44.4\%). A total of $312(32 \%)$ respondents preferred e-liquid that contained $\leq 6 \mathrm{mg}$ nicotine, followed by $212(21.7 \%)$ who were oblivious to the nicotine level (Table 2). About 178 (18.3\%) preferred e-liquid without nicotine, while only $2.4 \%$ preferred e-liquid with a high amount of nicotine ( $\geq 16 \mathrm{mg}$ ).

This study showed that over $67 \%$ of the respondents did not report any health impact and were not certain if they suffered any adverse effects from the use of e-cigarettes. Among the adverse effects experienced by the users were: dizziness $14.4 \%$, cough $14.1 \%$, headache $12.4 \%$, addiction to e-cigarette use $9.5 \%$, chest pain $6.9 \%$, and shortness of breath $5.7 \%$. Other adverse effects, experienced by less than $5 \%$ of the respondents, were: vomiting $4.5 \%$, decreased appetite $4.1 \%$, insomnia $4.0 \%$, weight loss $1.9 \%$, depression $1.6 \%$, and anxiety $1.1 \%$. Dual users had significantly experienced more adverse effects compared to exclusive e-cigarette users (Table 3 ).

Figure 2 depicts the feelings of respondents towards
Table 2. Respondents smoking e-cigarette-use profile

\begin{tabular}{|c|c|c|}
\hline Profiles & $\begin{array}{l}\text { Frequency } \\
\text { (in) }\end{array}$ & Per cent $(\%)$ \\
\hline \multicolumn{3}{|c|}{$\begin{array}{l}\text { Age of smoking initiation (years), } \\
n=1036\end{array}$} \\
\hline$<10$ & 39 & 3.8 \\
\hline $10-15$ & 380 & 36.7 \\
\hline $16-20$ & 569 & 54.9 \\
\hline $21-25$ & 48 & 4.6 \\
\hline \multicolumn{3}{|c|}{ Family member who smokes, $n=1284$} \\
\hline Yes & 679 & 53.5 \\
\hline No & 605 & 46.5 \\
\hline \multicolumn{3}{|c|}{ Average cigarettes per day, $n=1036$} \\
\hline Did not answer & 36 & 3.5 \\
\hline$\leq 5$ & 584 & 56.4 \\
\hline $6-10$ & 252 & 24.3 \\
\hline $11-15$ & 76 & 7.3 \\
\hline $16-20$ & 70 & 6.8 \\
\hline$\geq 21$ & 18 & 1.8 \\
\hline \multicolumn{3}{|c|}{ Preferable cigarette, $n=1036$} \\
\hline Standard & 791 & 76.4 \\
\hline Light & 279 & 26.9 \\
\hline
\end{tabular}


Table 2. Continued

\begin{tabular}{|c|c|c|c|c|c|}
\hline Profiles & $\begin{array}{c}\text { Frequency } \\
\text { (n) }\end{array}$ & Per cent $(\%)$ & Profiles & $\begin{array}{l}\text { Frequency } \\
\text { (In) }\end{array}$ & Per cent $(\%)$ \\
\hline Menthol & 218 & 21.0 & University student & 229 & 17.6 \\
\hline Tobacco (Indonesia) & 151 & 14.6 & Outside peers & 144 & 11.1 \\
\hline Cigar/Curut & 55 & 5.3 & Unsure & 187 & 14.4 \\
\hline Others (e.g. Harvest, John, U2, Mevius) & 37 & 3.6 & Number of e-cigarette devices, $n=506$ & & \\
\hline Source of e-cigarette information & & & 1 & 387 & 76.5 \\
\hline Family & 100 & 7.7 & 2 & 81 & 16.0 \\
\hline Product seller & 160 & 12.3 & 3 & 21 & 4.2 \\
\hline Advertisement & 189 & 14.5 & $\geq 4$ & 17 & 3.6 \\
\hline University student & 749 & 57.5 & Preferable e-juice, $n=975$ & & \\
\hline Internet & 476 & 36.6 & Local & 542 & 55.6 \\
\hline Magazines & 80 & 6.1 & Imported & 433 & 44.4 \\
\hline Outside peer & 488 & 37.5 & Types of e-juice, $n=975$ & & \\
\hline Media & 349 & 28.8 & Without nicotine (no flavours) & 178 & 18.3 \\
\hline Unsure & 127 & 9.8 & Without nicotine (with flavours)* & 85 & 8.7 \\
\hline Source of e-cigarettes & & & $\leq 6 \mathrm{mg}$ nicotine & 312 & 32.0 \\
\hline Wholesale & 109 & 8.4 & $\leq 9 \mathrm{mg}$ nicotine & 84 & 8.6 \\
\hline Booth seller & 135 & 10.4 & $\leq 12 \mathrm{mg}$ nicotine & 79 & 8.1 \\
\hline Family member & 32 & 2.5 & $\geq 16$ mg nicotine & 23 & 2.4 \\
\hline Retailer & 493 & 37.9 & Unsure & 212 & 21.7 \\
\hline Online & 193 & 14.8 & Unanswered & 2 & 0.2 \\
\hline Exchange among peers & 126 & 9.7 & *e.g. mango, mint, redbull, grape, vanilla. & & \\
\hline
\end{tabular}

Table 3. Adverse events occurrence based on types of cigarette e cigarette users

\begin{tabular}{|c|c|c|c|c|c|c|c|}
\hline \multirow[b]{2}{*}{ Variable } & \multicolumn{7}{|c|}{ Types of cigarette e cigarette users $(n, \%)$} \\
\hline & Dual user & $\begin{array}{l}\text { E cigarette user } \\
\text { (never smoker) }\end{array}$ & $\begin{array}{l}\text { E cigarette user } \\
\text { (ex smoker) }\end{array}$ & $\begin{array}{l}\text { Smoker (never } \\
\text { use e cigarette) }\end{array}$ & $\begin{array}{l}\text { Ex smoker } \\
\text { (never use } \\
\text { e cigaretle) }\end{array}$ & $\chi^{2}$ & $p$ \\
\hline \multicolumn{8}{|l|}{ Adverse events } \\
\hline None & 302 (33.0) & 175 (19.1) & $111(12.1)$ & 244 (26.7) & $83(9.1)$ & 193.488 & $<0.001^{*}$ \\
\hline 1 Symptom & $103(54.5)$ & 47 (24.9) & 39 (20.6) & $0(0.0)$ & $0(0.0)$ & & \\
\hline 2 Symptoms & 57 (58.8) & $21(21.6)$ & 19 (19.6) & $0(0.0)$ & $0(0.0)$ & & \\
\hline$\geq 3$ Symptoms & $63(62.4)$ & $23(22.8)$ & 15 (14.9) & $0(0.0)$ & $0(0.0)$ & & \\
\hline Total & $525(40.3)$ & 266 (20.4) & $184(14.1)$ & 244 (18.7) & $83(6.4)$ & & \\
\hline
\end{tabular}

${ }^{*}$ Chi-squared test. Values are expressed as frequency and per cent ( $\mathrm{n} \%$ ). $\mathrm{p}<0.05$ compares dual users, e-cigarette user (never smoker), e-cigarette user (ex-smoker), smoker (never use e-cigarette) and ex-smoker (never use e-cigarette).

e-cigarette use as an alternative to smoking cessation, where $31.9 \%$ felt uncertain about the best alternative means for quitting smoking, $29.5 \%$ did not mention any need for an alternative, while $28.2 \%$ agreed that e-cigarettes could be used as an alternative means for quitting smoking. Further, smoking cessation methods chosen were: nicotine lozenges $23.7 \%$, a support group $19.6 \%$, health counseling $16.6 \%$, nicotine patch $10.8 \%$, nicotine replacement drug $9.8 \%$, other $7.0 \%$, and traditional medicine $5.8 \%$.
With regards to the reason for e-cigarette use provided by dual users $(n=975), 64.6 \%$ of the respondents use e-cigarette due to their own desire, personal beliefs $64.6 \%$, social influence $54.8 \%$, emotional factors (boredom, loneliness and stress) $62.6 \%$, current trends $62.7 \%$, while $57.8 \%$ claimed to use e-cigarettes as a means for quitting smoking.

The study found that majority of the respondents were dual users, the high-risk group (involved in tobacco/nicotine use). The bivariate analysis revealed 
Figure 2. Students opinion on the most effective alternative for smoking cessation ( $\mathrm{n}=1036)$

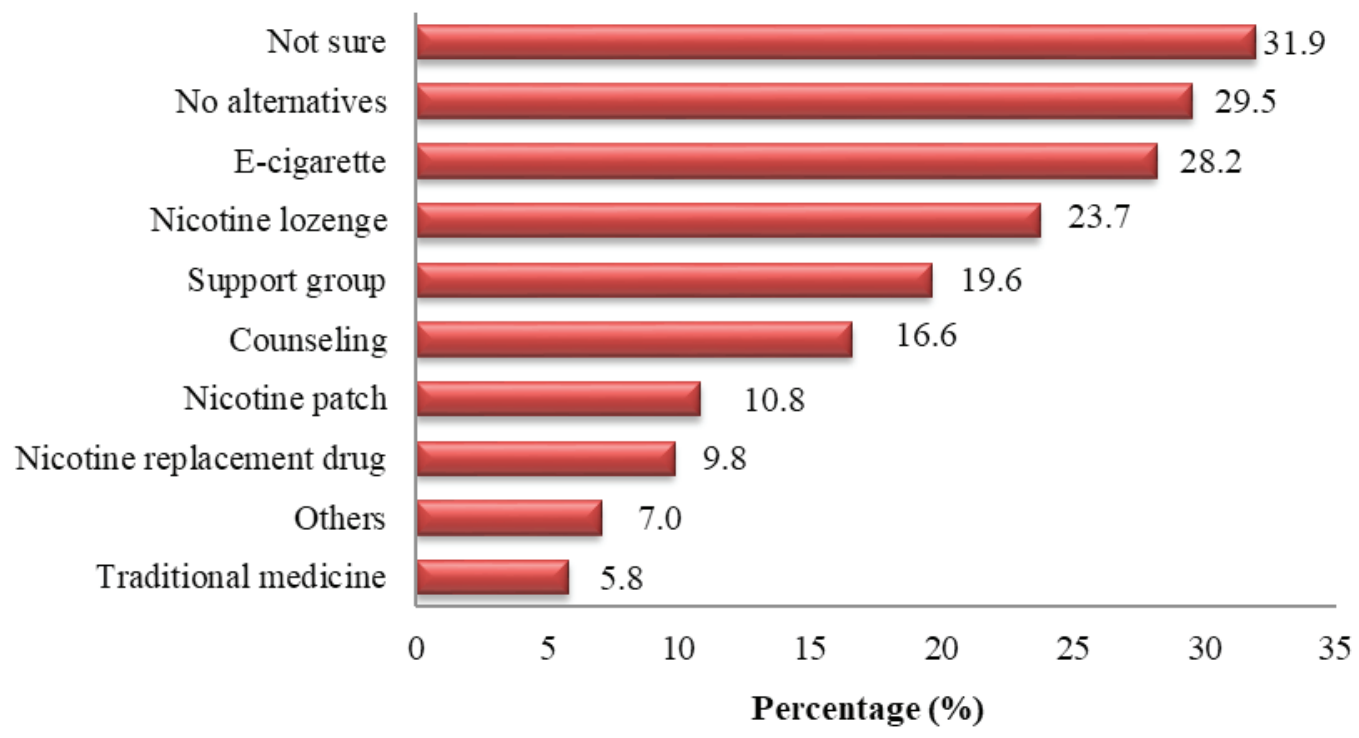

that gender as well as three reasons for e-cigarette use (own desire, personal belief, aim to quit smoking) have a significant relationship with e-cigarette use (Table 4). Of the 975 male e-cigarette users, $52.9 \%$

were dual users, while $42.3 \%$ from the same category of male respondents were exclusive e-cigarette users. Among the dual users' category, the reasons given for e-cigarette use were: own desire $37.8 \%$,

Table 4. Association between dual user and e cigarette (e-cig) users with sociodemographics, adverse events occurrence, comorbidity disease and reason for e cigarette use initiation among students (n=975)

\begin{tabular}{|c|c|c|c|c|c|c|c|}
\hline \multirow[b]{2}{*}{ Variable } & \multirow{2}{*}{$\begin{array}{c}\text { Dual user } \\
\text { n (\%) }\end{array}$} & \multirow{2}{*}{$\begin{array}{c}\text { E cig user } \\
\text { n }\left({ }^{\circ} \%\right)\end{array}$} & \multirow[b]{2}{*}{$x^{2}$} & \multirow[b]{2}{*}{$p$} & \multirow[b]{2}{*}{ OR } & \multicolumn{2}{|c|}{$95^{\circ} \circ \mathrm{CI}$} \\
\hline & & & & & & Lower & Upper \\
\hline \multicolumn{8}{|l|}{ Institution } \\
\hline Public & $277(28.4)$ & $226(23.2)$ & 0.63 & 0.43 & 1.11 & 0.86 & 1.42 \\
\hline Private & $248(25.4)$ & $224(23.0)$ & & & & & \\
\hline \multicolumn{8}{|l|}{ Age (years) } \\
\hline$\leq 21$ & $320(32.8)$ & $296(30.4)$ & 2.42 & 0.12 & 0.81 & 0.62 & 1.05 \\
\hline$>21$ & $205(21.0)$ & $154(15.8)$ & & & & & \\
\hline \multicolumn{8}{|l|}{ Gender } \\
\hline Male & $516(52.9)$ & 412 (42.3) & 23.92 & $<0.001^{*}$ & 0.19 & 0.09 & 0.40 \\
\hline Female & $9(0.9)$ & $38(3.9)$ & & & & & \\
\hline \multicolumn{8}{|l|}{ Ethnicity } \\
\hline Malay & $400(41.0)$ & $350(35.9)$ & 0.34 & 0.56 & 1.09 & 0.81 & 1.48 \\
\hline Others & $125(12.8)$ & $100(10.3)$ & & & & & \\
\hline \multicolumn{8}{|c|}{ Marital status } \\
\hline Single & $516(52.9)$ & 441 (45.2) & 0.11 & 0.74 & 0.85 & 0.34 & 2.17 \\
\hline Married & $9(0.9)$ & $9(0.9)$ & & & & & \\
\hline \multicolumn{8}{|c|}{ Level of education } \\
\hline Degree & $351(36.0)$ & $280(28.7)$ & 2.28 & 0.13 & 0.82 & 0.63 & 1.06 \\
\hline Others & $174(17.8)$ & $170(17.4)$ & & & & & \\
\hline \multicolumn{8}{|c|}{ Household income (MYR/month) } \\
\hline$\leq \mathrm{RM} 4000$ & $228(27.8)$ & $206(25.1)$ & 0.01 & 0.93 & 0.99 & 0.75 & 1.30 \\
\hline$>$ RM4000 & $204(24.9)$ & $182(22.2)$ & & & & & \\
\hline
\end{tabular}


Table 4. Continued

\begin{tabular}{|c|c|c|c|c|c|c|c|}
\hline \multirow[b]{2}{*}{ Varriable } & \multirow{2}{*}{$\begin{array}{c}\text { Dual user } \\
\text { n }(\%)\end{array}$} & \multirow{2}{*}{$\begin{array}{c}\text { E cig user } \\
\text { n }(\%)\end{array}$} & \multirow[b]{2}{*}{$x^{2}$} & \multirow[b]{2}{*}{$p$} & \multirow[b]{2}{*}{ OR } & \multicolumn{2}{|c|}{$95 \%$ CI } \\
\hline & & & & & & Lower & Upper \\
\hline \multicolumn{8}{|c|}{ Adverse events occurrence } \\
\hline$\leq 2$ symptoms & $462(47.4)$ & $412(42.3)$ & 3.30 & 0.07 & 0.68 & 0.44 & 1.03 \\
\hline >2 symptoms & $63(6.5)$ & $38(3.9)$ & & & & & \\
\hline \multicolumn{8}{|l|}{ Comorbidity disease } \\
\hline None (healthy) & $484(49.6)$ & $407(41.7)$ & 0.94 & 0.33 & 1.25 & 0.80 & 1.95 \\
\hline Having comorbidity & $41(4.2)$ & $43(4.4)$ & & & & & \\
\hline \multicolumn{8}{|c|}{ Reason for e-cigarette use } \\
\hline \multicolumn{8}{|l|}{ i) Own desire } \\
\hline Low & $156(16.0)$ & 189 (19.4) & 16.00 & $<0.001^{*}$ & 0.58 & 0.45 & 0.76 \\
\hline High & 369 (37.8) & $261(26.8)$ & & & & & \\
\hline \multicolumn{8}{|l|}{ ii) Social influence } \\
\hline Low & $236(24.2)$ & $205(21.0)$ & 0.04 & 0.85 & 0.98 & 0.76 & 1.26 \\
\hline High & $289(29.6)$ & $245(25.1)$ & & & & & \\
\hline \multicolumn{8}{|l|}{ iii) Personal belief } \\
\hline Low & $162(16.6)$ & $184(18.9)$ & 10.65 & $<0.001^{*}$ & 0.64 & 0.50 & 0.84 \\
\hline High & $363(37.2)$ & $266(27.3)$ & & & & & \\
\hline \multicolumn{8}{|l|}{ iv) Currents trends } \\
\hline Low & $195(20.0)$ & 169 (17.3) & 0.02 & 0.89 & 0.98 & 0.76 & 1.27 \\
\hline High & 330 (33.8) & $281(28.8)$ & & & & & \\
\hline \multicolumn{8}{|c|}{ v) Aim to quit smoking } \\
\hline Low & $190(19.5)$ & $221(22.7)$ & 16.59 & $<0.001^{*}$ & 0.59 & 0.45 & 0.76 \\
\hline High & $335(34.4)$ & $229(23.5)$ & & & & & \\
\hline \multicolumn{8}{|l|}{ vi) Self emotion } \\
\hline Low & $186(19.1)$ & $179(18.4)$ & 1.96 & 0.16 & 0.83 & 0.64 & 1.08 \\
\hline High & $339(34.8)$ & $271(27.8)$ & & & & & \\
\hline
\end{tabular}

Data exclude non-e-cigarette user with analysis using chi-squared test. E-cigarette user refers to exclusive e-cigarette user. $0 \mathrm{R}$ : odds ratio, $\mathrm{Cl}$ : confidence interval. ${ }^{*} \mathrm{p}<0.05$ indicates significant difference does exist.

personal belief $37.2 \%$, mood disorder $34.8 \%$, aim to quit smoking $34.4 \%$, current trend $33.8 \%$, and social influence $29.6 \%$. Among the exclusive e-cigarette users' category, main reasons given for e-cigarette use were: current trend $28.8 \%$, mood disorder $27.8 \%$, personal belief $27.3 \%$, own desire $26.8 \%$, social influence $25.1 \%$, and aim to quit smoking $23.5 \%$.

Logistic regression was performed to ascertain the impact of age, gender, ethnicity, level of education, adverse events occurrence, comorbidity disease and the reason for e-cigarette use. Exclusive e-cigarette use was significantly associated with gender $(\mathrm{AOR}=0.19,95 \% \mathrm{CI}: 0.09-0.40, \mathrm{p}<0.001)$ and own desire $(\mathrm{AOR}=0.58,95 \% \mathrm{CI}: 0.45-0.76, \mathrm{p}<0.001)$, personal belief (AOR $=0.64,95 \%$ CI: $0.50-0.84$, $\mathrm{p}=0.001)$ and aim to quit smoking $(\mathrm{AOR}=0.59,95 \%$ CI: $0.45-0.76, \mathrm{p}<0.001)$. However, the multivariate analysis showed that exclusive e-cigarette use was only associated with gender $(\mathrm{p}<0.001)$ with an adjusted odds ratio of becoming e-cigarette user 0.18 times lower for males compared to females (Table 5).

Table 5. Factors associated with e-cigarette users in univariate and multivariate analysis among students (n=975)

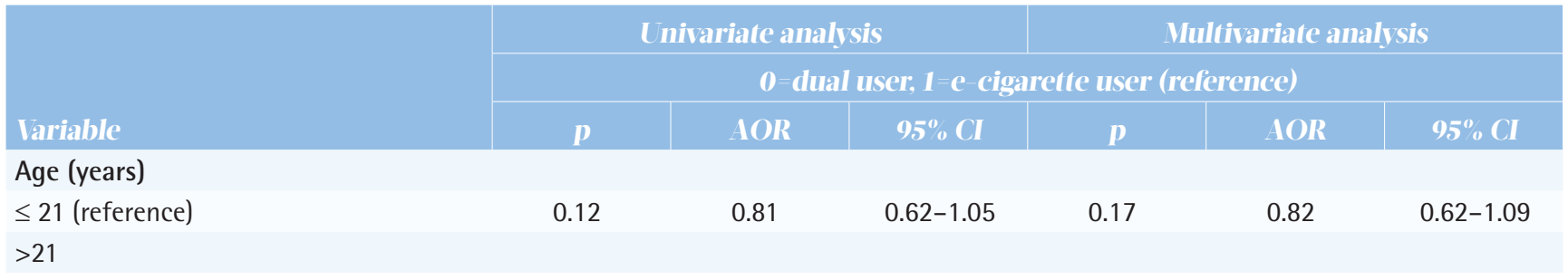


Table 5 . Continued

\begin{tabular}{|c|c|c|c|c|c|c|}
\hline \multirow[b]{3}{*}{ Variable } & \multicolumn{3}{|c|}{ Univariate analysis } & \multicolumn{3}{|c|}{ Vultivariate analysis } \\
\hline & \multicolumn{6}{|c|}{ (1) dual user; I e cigarette user (reference) } \\
\hline & $p$ & AOR & $95^{\circ} \circ \mathrm{CI}$ & p & AOR & $95^{\circ}$ 。 CI \\
\hline \multicolumn{7}{|l|}{ Gender } \\
\hline Female (reference) & $<0.001^{*}$ & 0.19 & $0.09-0.40$ & $<0.001^{*}$ & 0.18 & $0.09-0.39$ \\
\hline \multicolumn{7}{|l|}{ Male } \\
\hline \multicolumn{7}{|l|}{ Ethnicity } \\
\hline Others (reference) & 0.56 & 1.09 & $0.81-1.48$ & 0.17 & 1.24 & $0.91-1.71$ \\
\hline \multicolumn{7}{|l|}{ Malay } \\
\hline \multicolumn{7}{|l|}{ Level of education } \\
\hline Others (reference) & 0.13 & 0.82 & $0.63-1.06$ & 0.44 & 0.89 & $0.67-1.19$ \\
\hline \multicolumn{7}{|l|}{ Degree } \\
\hline \multicolumn{7}{|l|}{ Adverse events occurrence } \\
\hline$\leq 2$ symptoms (reference) & 0.07 & 0.68 & $0.44-1.03$ & 0.05 & 0.65 & $0.42-1.01$ \\
\hline \multicolumn{7}{|l|}{$>2$ symptoms } \\
\hline \multicolumn{7}{|l|}{ Comorbidity disease } \\
\hline None (reference) & 0.33 & 1.25 & $0.80-1.9$ & 0.29 & 1.29 & $0.81-2.05$ \\
\hline \multicolumn{7}{|l|}{ Having comorbidity } \\
\hline \multicolumn{7}{|l|}{ Reason for e-cigarette use } \\
\hline \multicolumn{7}{|l|}{ i) Own desire } \\
\hline Low (reference) & $<0.001^{*}$ & 0.58 & $0.45-0.76$ & 0.27 & 0.82 & $0.59-1.16$ \\
\hline \multicolumn{7}{|l|}{ High } \\
\hline \multicolumn{7}{|l|}{ ii) Personal belief } \\
\hline Low (reference) & $0.001^{*}$ & 0.64 & $0.50-0.84$ & 0.34 & 0.85 & $0.61-1.19$ \\
\hline \multicolumn{7}{|l|}{ High } \\
\hline \multicolumn{7}{|l|}{ iii) Aim to quit smoking } \\
\hline Low (reference) & $<0.001^{*}$ & 0.59 & $0.45-0.76$ & 0.06 & 0.72 & $0.51-1.01$ \\
\hline High & & & & & & \\
\hline
\end{tabular}

Data exclude non-e-cigarette user with analysis using logistic regression. E-cigarette user refers to exclusive e-cigarette user. AOR: adjusted odds ratio, Cl: confidence interval. ${ }^{*} p<0.05$ indicates significant difference does exist.

\section{DISCUSSION}

The study assessed factors related to e-cigarette use through which user profiles were created. The study revealed that male respondents were the majority e-cigarette users, most of them young and residing in urban areas. The respondents smoked cigarettes alongside their family members, colleagues and peers who either smoked tobacco, used an e-cigarette, or both. This finding is similar to the e-cigarette user profile reported by Jun et al. ${ }^{20}$ and Joan-Carles et al. ${ }^{21}$. In Malaysia, smoking among females is culturally unacceptable, which affirms the low number of female e-cigarette users found in the study. Young people have strong desires to try something new; living in an urban settings allows easy access to e-cigarette sources and living in proximity to a smoking environment enhances smoking interest. Involvement with e-cigarette use exposes the users to various health risks, particularly those who use it as an alternative to quitting cigarette smoking. The implication here is the possible lack of knowledge about smoking quitting methods, despite the availability of alternative means for quitting cigarette smoking, such as nicotine lozenges, support group, counselling, nicotine patch, nicotine replacement drug and traditional medicine. The listed methods are safe and subsidized by the government, but were only used by $25 \%$ of the respondents.

In this study, about $74.8 \%$ of the respondents were e-cigarette users, the finding is augmented by the Saddleson et al. ${ }^{36}$ assertion that most university/ college students use e-cigarettes for pleasure. However, a study conducted in France found that $70 \%$ of college students in two major campuses never used 
e-cigarette ${ }^{37}$. Also, some studies in the United States and other countries show that e-cigarette users may as well use conventional cigarettes ${ }^{38,39}$ and drugs ${ }^{40}$ in the future. It is also worrying that e-cigarette use among ex-smokers may cause a return to cigarette smoking ${ }^{41}$.

Over three-quarters of the respondents were involved in smoking, including ex-smokers. Of them, $54.9 \%$ started smoking at the age of $16-20$ years, when they were in high school or just entering university. This situation clearly indicates that strategic prevention planning should target this age group and younger. Rigotti ${ }^{42}$ stated that university students should be targeted for behavior change advocacy, because adolescents were the main marketing target group of the tobacco industry. It was further revealed that $34.5 \%$ of the respondents were exclusive e-cigarette users, with preference to e-juice without nicotine (only flavors) and lower level of nicotine $(\leq 6 \mathrm{mg} \text { of nicotine })^{41}$.

Health effects of smoking are well known due to the existence of evidence-based research outcomes and established linkage to cancer, heart disease, and stroke. Information on smoking hazards are available and easily accessible through various media outlets (e.g. posters, advertisements on buildings and vehicles, video, radio and television ads etc.). Despite this development for a conventional cigarette, information on e-cigarette adverse effects are relatively new and emerging, and in need of more research. In this study, adverse effects like dizziness, cough, headache, addiction and chest pain were reported. Findings by Hua et al. ${ }^{43}$ posit that there are over 405 different health-related effects experienced by users. Additionally, most common health-related effects occurred in the respiratory, neurological, sensory and digestive systems, while direct health effects occurred solely in the respiratory system ${ }^{43}$.

E-cigarette marketers often advertise it as a safe and healthier alternative to conventional smoking and that it aids smoking cessation ${ }^{4-48}$. The study found that e-cigarettes were used by the respondents for various reasons: own desire, personal beliefs, selfemotion, and as a current smoking trend. Using it as a means for quitting smoking is popular among dual users $(59.4 \%)$. Chapman et al. ${ }^{49}$ stated that e-cigarette use is not consistent with attempting to quit tobacco smoking among young adults, as adults most often report e-cigarette use as a substitute for tobacco and not as a means for quitting ${ }^{24,25,49}$.

The effectiveness of e-cigarette use as a smoking cessation tool is unclear ${ }^{1,4,20}$, and subject for further research. Moreover, e-cigarette use is not without risk, but much less dangerous than tobacco, as it has less carcinogenic chemicals (e.g. acetone, acroline, benzene, cadmium, carbon monoxide, toluene, etc. $)^{46,48}$. Lynn et al. ${ }^{50}$ suggested that the mistaken perception of lesser risk may be the influencing factor for e-cigarette use as a substitute for tobacco smoking. Further research on the health effects of e-cigarette use should be conducted, to ascertain its severity on human health.

The main limitation of this study is that the data were only collected in one of the States in Malaysia within a certain time duration. Therefore, the samples do not represent a national proportion and hence have limited generalizability. Also, the self-reporting mechanism used in data collection could result in a biased response. Despite these limitations, the findings present a good profile of e-cigarette users and a framework with which to guide decision making and future research on e-cigarette use. Additionally, e-cigarette use was more popular among young people in this study, larger studies in diverse locations and populations might address the challenges of this study.

\section{CONCLUSIONS}

The study provides evidence-based information for a behaviour change campaign on e-cigarette use among university students. Both cigarettes, as well as e-cigarette use among students, for whatever reason, need to be discouraged. Achieving meaningful mitigation of the use of tobacco products needs the combined efforts of academics, civil society, government, industry and communities to find effective means for a solution to the tobacco epidemic.

\section{REFERENCES}

1. Gravely S, Fong GT, Cummings KM, et al. Correction: Gravely, S., et al. Awareness, Trial, and Current Use of Electronic Cigarettes in 10 Countries: Findings from the ITC Project. Int. J. Environ. Res. Public Health 2014, 11, 11691-11704. Int. J. Environ. Res. Public Health. 2015;12(5):4631-4637. doi:10.3390/ijerph120504631

2. Caponnetto P, Campagna D, Papale GM, Russo C, Polosa R. The emerging phenomenon of electronic cigarettes. Expert Rev Respir Med. 2012;6(1):63-74. doi:10.1586/ers.11.92

3. Dockrell M, Morrison R, Bauld L, McNeill Ann. E-cigarettes: Prevalence and attitudes in Great Britain. Nicotine Tob Res. 


\section{3;15:1737-1744. doi:10.1093/ntr/ntt057}

4. King BA, Patel R, Nguyen KH, Dube SR. Trends in awareness and use of electronic cigarettes among U.S. adults, 2010-2013. Nicotine Tob. Res. 2015;17:219-227. doi:10.1093/ntr/ntu191

5. Farsalinos KE, Poulas K, Voudris V, Le Houezec J. Electronic cigarette use in the European Union: Analysis of a representative sample of 27,460 Europeans from 28 countries. Addiction. 2016;111:2032-2040. doi:10.1111/add.13506

6. Weaver SR, Majeed BA, Pechacek TF, Nyman AL, Gregory KR, Eriksen MP. Use of electronic nicotine delivery systems and other tobacco products among USA adults, 2014: Results from a national survey. Int. J. Public Health. 2016;61:177-188. doi:10.1007/s00038-015-0761-0

7. World Health Organization. WHO Study Group on Tobacco Product Regulation. Report on the Scientific Basis of Tobacco Product Regulation. WHO Technical Report Series, no. 955. http://www.who.int/tobacco/ global_interaction/tobreg/publications/tsr_955/en/. Published January, 2010. Accessed July 25, 2018.

8. Schmid T. Asia's E-Cig Regulations: Confusion \& Uncertainty. Tobacco Asia. http://www. tobaccoasia.com/ features/asia-e-cig-regulations-confusion-uncertainty/. Published May, 2016. Accessed July 28, 2018.

9. Alex Frew McMillan. Vaping in Asia Could Save Big Tobacco. https://www.thestreet.com/story/14273799/1/ vaping-in-asia-could-save-big-tobacco.html. Published August, 2017. Accessed July 28, 2018.

10. Palipudi KM, Mbulo L, Morton J, et al. Awareness and Current Use of Electronic Cigarettes in Indonesia, Malaysia, Qatar, and Greece: Findings From 2011-2013 Global Adult Tobacco Surveys. Nicotine \& Tobacco Research. 2016;18:501. doi:10.1093/ntr/ntv081

11. World Health Organization. Global Adult Tobacco Survey (GATS): Fact Sheet Philiphines 2015. www.who.int/ tobacco/surveillance/survey/gats/phl_country_report. pdf. Published February, 2017. Accessed July 25, 2018.

12. World Health Organization. Global Adult Tobacco Survey (GATS): Fact Sheet Vietnam 2015. http://www.who.int/ tobacco/surveillance/survey/gats/VN-2015_FactSheet_ Standalone _ E_Oct2016.pdf?ua=1. Published August, 2017. Accessed July 25, 2018.

13. World Health Organization. Global Youth Tobacco Survey (GYTS): Fact Sheet Cambodia 2016. https:// nccd.cdc.gov/GTSSDataSurveyResources/Ancillary/ DownloadAttachment.asp $\mathrm{x}$ ?ID $=3378$. Published December, 2017. Accessed July 25, 2018.

14. World Health Organization. Global Youth Tobacco Survey (GYTS): Fact Sheet Myanmar 2016. http://www.searo. who.int/tobacco/data/mmr_gyts_2016_fs.pdf?ua=1. Published December, 2017. Accessed July 25, 2018.

15. Ab Rahman J, Nik Mohamed MH, Kartiwi M, et al. National E-cigarette Survey (NECS) 2016 in Malaysia Method and population characteristics. Med J Malaysia.
2017;72(Suppl.1):A143

16. Cohen A. How Many People Vape Around The World? vaping.com https://vaping.com/blog/data/how-manypeople-vape-around-the-world/. Published November, 2017. Accessed July 28, 2018.

17. Wong LP, Mohamad Shakir SM, Alias H, Aghamohammadi $\mathrm{N}$, Hoe VC. Reasons for using electronic cigarettes and intentions to quit among electronic cigarette users in Malaysia. J Community Health. 2016;41:1101-1109. doi:10.1007/s10900-016-0196-4

18. Jusoh I. Vape ban in all public universities. Malay Mail. November 22, 2015. http://www.themalaymailonline.com/ malaysia/article/idris-jusoh-vape-ban-in-all-public-universities. Published November, 2015. Assessed April 2, 2017.

19. Private varsities ban smoking and Vaping. The Star Online. December 2, 2015. http://www.thestar.com.my/news/ nation/2015/12/02/private-varsities-ban-smoking-andvaping/. Published December, 2015. Assessed April 2, 2017.

20. Goniewicz ML, Zielinska-Danch W. Electronic cigarette use among teenagers and young adults in Poland. Pediatrics. 2012;130(4):e879-e885. doi:10.1542/peds.2011-3448

21. Cho JH, Shin E, Moon SS. Electronic-cigarette smoking experience among adolescents. Journal of Adolescent Health. 2011;49:542-546. doi:10.1016/j.jadohealth.2011.08.001

22. Suris JC, Berchtold A, Akre C. Reasons to use e-cigarettes and associations with other substances among adolescents in Switzerland. Drug Alcohol Depend. 2015;153:140-144. doi:10.1016/j.drugalcdep.2015.05.034

23. Babineau K, Taylor K, Clancy L. Electronic Cigarette Use among Irish Youth: A Cross Sectional Study of Prevalence and Associated Factors. PLoS ONE. 2015;10(5):e0126419. doi:10.1371/journal.pone.0126419

24. Etter JF, Bullen C. Electronic cigarette: users profile, utilization, satisfaction and perceived efficacy. Addiction. 2011;106(11):2017-2028. doi:10.1111/j.1360-0443.2011.03505.x

25. Etter JF. Electronic cigarettes: A survey of users. BMC Public Health. 2010;10:231. doi:10.1186/1471-2458-10-231

26. Schmidt L, Reidmohr A, Harwell TS, Helgerson SD. Prevalence and reasons for initiating use of electronic cigarettes among adults in Montana, 2013. Preventing Chronic Disease. 2014;11:E204. doi:10.5888/pcd11.140283

27. Biener L, Hargraves JL. A longitudinal study of electronic cigarette use among a population-based sample of adult smokers: Association with smoking cessation and motivation to quit. Nicotine Tob Res. 2015;17(2):127133. doi:10.1093/ntr/ntu200

28. Kong G, Morean ME, Cavallo DA, Camenga DR, Krishnan-Sarin S. Reasons for electronic cigarette experimentation and discontinuation among adolescents and young adults. Nicotine Tob Res. 2015;17(7):847-854. doi:10.1093/ntr/ntu257

29. McDonald EA, Ling PM. One of several 'toys' for smoking: young adult experiences with electronic cigarettes in 
New York City. Tob Control. 2015;24(6):588-593. doi:10.1136/tobaccocontrol-2014-051743

30. Harrell MB, Weaver SR, Loukas A, et al. Flavoured e-cigarette use: Charachterizing youth, young adult, and adult users. Preventive Medicine Report. 2017;5:33-40. doi:10.1016/j.pmedr.2016.11.001

31. Ambrose BK, Day HR, Rostron B. Flavored tobacco product use among US youth aged 12-17 years, 2013-2014. JAMA. 2015;314:1871-1873. doi:10.1001/jama.2015.13802

32. Choi K, Fabian L, Mottey N, Corbett A, Forster J. Young adults' favorable perceptions of snus, dissolvable tobacco products, and electronic cigarettes: Findings from a focus group study. American Journal of Public Health. 2012;102(11):2088-2093. doi:10.2105/AJPH.2011.300525

33. Dutra LM, Glantz SA. Electronic cigarettes and conventional cigarettes use among US Adolescents: A cross-sectional study. JAMA Pediatricts. 2014;168(7):610617. doi:10.1001/jamapediatrics.2013.5488

34. Pepper JK, Ribisl KM, Emery SL, Brewer NT. Reasons for starting and stopping electronic cigarette use. Int J Environ Res Public Health. 2014;11:10345-10361. doi:10.3390/ijerph111010345

35. American Heart Association. Electronic cigarettes: A policy statement from the American Heart Association. Dallas. http://circ.ahajournals.org/content/130/16/1418.full.pdf+ html. Published October, 2014. Assessed April 8, 2017.

36. Saddleson ML, Kozlowski LT, Giovino GA, et al. Risky behaviors, e-cigarette use and susceptibility of use among college students. Drug \& Alcohol Dependence. 2015;149:25-30. doi:10.1016/j.drugalcdep.2015.01.001

37. Tavolacci MP, Vasiliu A, Romo L, Kotbagi G, Kern L, Ladner J. Patterns of electronic cigarette use in current and ever users among college students in France: A cross-sectional study. BMJ Open. 2016;6:e011344. doi:10.1136/bmjopen-2016-011344

38. Leventhal AM, Strong DR, Kirkpatrick MG, et al. Association of electronic cigarette use with initiation of combustible tobacco product smoking in early adolescence. JAMA. 2015;314(7):700-707. doi:10.1001/jama.2015.8950

39. Wills TA, Knight R, Williams RJ, Pagano I, Sargent JD. Risk factors for exclusive e-cigarette use and dual e-cigarette use and tobacco use in adolescents. Pediatrics. 2015;135(1):e43-51. doi:10.1542/peds.2014-0760

40. Newman K. NIH Survey: Students vaping, using Marijuana more in 2017. Teens' use of vaporizers and marijuana could lead to future drug habits researchers warn. U.S. News \& World Report. https://www.usnews. com/news/national-news/articles/2017-12-14/nihsurvey-alcohol-marijuana-and-vaping-top-students-2017drug-use. Published, 2017. Assessed April 8, 2017.

41. Sinniah D, Khoo EJ. E-cigarettes: Facts and legal status. IeJSME. 2015;9(3):10-19.

42. Rigotti NA. E-Cigarette use and subsequent tobacco use by adolescents: New evidence about a potential risk of e-cigarettes. JAMA. 2015;314(7):673-674. doi:10.1001/jama.2015.8382

43. Hua M, Alfi M, Talbot P. Health related effects reported by electronic cigarette users in online forums. Journal of Medical Internet Research. 2013;15:e59. doi:10.2196/jmir.2324

44. Palazzolo DL. Electronic Cigarettes and Vaping: A New Challenge in Clinical Medicine and Public Health. A Literature Review. Frontiers in Public Health. 2013;1:56. doi:10.3389/fpubh.2013.00056

45. Manuel. Choosing the lesser of the two evils. OLEIA. https:// www.oleia.net/choosing-lesser-two-evils-vaping-vs-cigarettetobacco/. Published August, 2017. Assessed October 14, 2017.

46. McNeill A, Hajek P. Underpinning evidence for the estimate that e-cigarette use is around 95\% safer than smoking. Authors' note. London, United Kingdom: Public Health England publications gateway; 2015.

47. GOV.UK. E-cigarettes around 95\% less harmful than tobacco estimates landmark review. https://www.gov.uk/ government/news/e-cigarettes-around-95-less-harmfulthan-tobacco-estimates-landmark-review. Published August, 2015. Assessed April 7, 2017.

48. Farsalinos KE, Polosa R. Safety evaluation and risk assessment of electronic cigarettes as tobacco cigarette substitutes: A systematic review. Therapeutic Advances in Drug Safety. 2014;5(2):67-86.doi:10.1177/2042098614524430

49. Carroll Chapman SL, Wu LT. E-Cigarette prevalence and correlates of use among adolescents versus adults: A review and comparison. Journal of Psychiatric Research. 2014;54:43-54. doi:10.1016/j.jpsychires.2014.03.005

50. Kozlowski LT, Homish DL, Homish GG. Daily users compared to less frequent users find vape as or more satisfying and less dangerous than cigarettes, and are likelier to use non-cig-alike vaping products. Preventive Medicine Reports. 2017;6:111-114. doi:10.1016/j.pmedr.2017.02.026

\section{ACKNOWLEDGEMENTS}

This work was produced under the Higher Education Policy Studies (HEPS) programme of the Ministry of Higher Education, Malaysia (MOHE). We thank all the Institutes and participants for their valuable contributions to this study. Our greatest appreciation to the Dean, Faculty of Medicine, Universiti Kebangsaan Malaysia, for permission to conduct this study. This manuscript was improved by the reviewers.

\section{CONFLICTS OF INTEREST}

The authors declare that they have no competing interests, financial or otherwise, related to the current work. All authors have completed and submitted the ICMJE Form for Disclosure of Potential Conflicts of Interest and report grants from Ministry of Higher Education Malaysia (MOHE), during the conduct of the study.

FUNDING

This work was supported by the Ministry of Higher Education, Malaysia (MOHE) through research grants (project code: FF-2016-301). The sponsor had no role in the designing, conducting, data analyzing and manuscript writing of this study.

PROVENANCE AND PEER REVIEW

Not commissioned; externally peer reviewed. 